\title{
DGMRES method augmented with eigenvectors for computing the Drazin-inverse solution of singular linear systems
}

\author{
MENG Bin \\ College of Science, Nanjing University of Aeronautics and Astronautics, \\ Nanjing 210016, People's Republic of China \\ E-mail: b.meng@nuaa.edu.cn
}

\begin{abstract}
The DGMRES method for solving Drazin-inverse solution of singular linear systems is generally used with restarting. But the restarting often slows down the convergence and DGMRES often stagnates. We show that adding some eigenvectors to the subspace can improve the convergence just like the method proposed by R.Morgan in [R.Morgan, A restarted GMRES method augmented with eigenvectors, SIAM J.Matrix Anal.Appl. 16 (1995)1154-1171]. We derive the implementation of this method and present some numerical examples to show the advantages of this method.
\end{abstract}

Mathematics Subject Classification. 65F15, 15A18

Key words and phrases. Drazin-inverse; DGMRES; Krylov subspace; Iterative method; Eigenvector.

\section{Introduction}

DGMRES is a new iterative method for computing the Drazin-inverse solution of linear systems [3]. Consider the linear system

$$
A x=b
$$

where $A \in \mathbb{C}^{n \times n}$ is a singular matrix. We recall that the Drazin-inverse solution of (11) is the vector $A^{D} b$, where $A^{D}$ is the Drazin-inverse of the singular matrix A.

In [2], A.Sidi proposed a general approach to Krylov subspace methods for computing Drazin-inverse solution. In that paper the authors do not put any restriction on $A$, that is, A is non-hermitian or hermitian, index of $A$ is arbitrary and the spectrum of A can have any shape. In [3], A.Sidi gave one of the Krylov subspace method named DGMRES, which is a GMRES-like algorithm. Like GMRES, in practical use, we often propose restarted DGMRES which denoted by DGMRES(m). DGMRES(m) is an economical computing 
and storgewise method for the Drazin-inverse solution. But restarting slows down the convergence and often stagnates (see [4, [5]). In the present paper we propose a DGMRES method augmented with eigenvectors, which can accelerate the convergence and overcome the stagnation. Classical GMRES augmented with eigenvectors was studied by R.Morgan (see [1]). Now we derive a DGMRES augmented with eigenvectors similarly. We give the convergence analysis of DGMRES augmented with eigenvectors which shows this method is more effective than DGMRES without augment. Then some numerical experiments are presented to show the convergence rate of DGMRES augmented with eigenvectors is remarkably improved, especially when the matrix has a very large or small nonzero eigenvalue.

The paper is organized as follows. In section 2, we will give a brief review of DGMRES. In section 3, we obtain the convergence analysis of DGMRES augmented with eigenvectors and derive the algorithm. In section 4, we present some numerical examples to compare the DGMRES with the DGMRES augmented with eigenvectors.

\section{DGMRES}

Throughout this paper, we suppose the index of $A$ is known. The index of $A$, denoted by $\operatorname{ind}(A)$, is the size of the largest Jordan block corresponding to the zero eigenvalue of $A$. DGMRES is a Krylov subspace methods for computing the Drazin-inverse solution $A^{D} b$. For more details we refer the readers to [2, [3].

We start with a initial vector $x_{0}$ and the method we interested in is to generate a sequence of vectors $x_{1}, x_{2}, \cdots$, which satisfies

$$
x_{m}=x_{0}+q_{m-1}(A) r_{0}, \quad r_{0}=: b-A x_{0},
$$

where $q_{m-1}(\lambda)=\sum_{i=1}^{m-a} c_{i} \lambda^{a+i-1}(a:=\operatorname{ind}(A))$. Then

$$
r_{m}:=b-A x_{m}=p_{m}(A) r_{0}
$$

where $p_{m}(\lambda)=1-\lambda q_{m-1}(\lambda)=1-\sum_{i=1}^{m-a} c_{i} \lambda^{a+i}$.

The Krylov subspace we will use is

$$
K_{m}\left(A ; A^{a} r_{0}\right)=\operatorname{span}\left\{A^{a} r_{0}, A^{a+1} r_{0}, \cdots, A^{m-1} r_{0}\right\}
$$

. The vector $x_{m}$ produced by DGMRES satisfies

$$
\left\|A^{a} r_{m}\right\|=\min _{x \in x_{0}+K_{m}\left(A ; A^{a} r_{0}\right)}\left\|A^{a}(b-A x)\right\| .
$$

Now we give restarting DGMRES Algorithm DGMRES(m).

Algorithm 1 DGMRES $(m)$

1. Choose initial guess $x_{0}$, and compute $r_{0}=b-A x_{0}$ and $A^{a} r_{0}$; 
2. Compute $\beta=\left\|A^{a} r_{0}\right\|$ and set $v_{1}=\beta^{-1} A^{a} r_{0}$;

3.Orthogonalize the vectors: $A^{a} r_{0}, A^{a+1} r_{0}, \cdots, A^{m} r_{0}$ by Arnoldi-Gram-Schmidt process.

For $j=1,2, \cdots$ do

For $i=1,2, \cdots$ do

Compute $h_{i, j}=\left\langle A V_{j}, V_{i}\right\rangle$.

Compute $\widehat{v}_{j}=A v_{j}-\sum_{i=1}^{j} h_{i, j} v_{i}$.

Set $h_{j+1, j}=\left\|\widehat{v}_{i}\right\|$ and $v_{j+1}=\widehat{v}_{j} / h_{j+1, j}$.

4. For $k=1,2, \cdots$, , form the matrices $\widehat{V}_{k} \in \mathbb{C}^{n \times k}$ and $\bar{H}_{k} \in \mathbb{C}^{(k+1) \times k}$.

$$
\begin{gathered}
\widehat{V}_{k}=\left[v_{1}, v_{2}, \cdots, v_{k}\right] ; \\
\bar{H}_{k}=\left(\begin{array}{cccc}
h_{11} & h_{12} & \cdots & h_{1 k} \\
h_{21} & h_{22} & \cdots & h_{2 k} \\
0 & h_{32} & \cdots & h_{3 k} \\
& & \cdots & \\
\vdots & \ddots & \ddots & \vdots \\
0 & \cdots & 0 & h_{k+1, k}
\end{array}\right)
\end{gathered}
$$

5. For $m=a+1, \cdots$, form the matrix $\widehat{H}_{m}=\bar{H}_{m} \bar{H}_{m-1} \cdots \bar{H}_{m-a}$.

6. Compute the $Q R$ factorization of $\widehat{H}_{m}: \widehat{H}_{m}=Q_{m} R_{m}$, where $R_{m}$ is upper triangular.

7. Solve the system $R_{m} y_{m}=\beta\left(Q_{m}^{*} e_{1}\right)$, where $e_{1}=(1,0, \cdots, 0)^{T}$.

8. Compute $x_{m}=x_{0}+\widehat{V}_{m-a} y_{m}$.

9. Restart: if $r_{m}=b-A x_{m}$ satisfied with the residual norm then stop, else let $x_{0}=x_{m}$ and go to 2 .

In 2, 3, the convergence analysis of DGMRES is given as follows.

Lemma 2 [2, 3] Denote the spectrum of $A$ by $\sigma(A)$ and choose $\Omega$ to be a closed domain in the $\lambda$-plane that contains $\sigma(A) \backslash\{0\}$ but not $\lambda=0$, such that its boundary is twice differentiable with respect to arc-length. Denote by $\Phi(\lambda)$ the conformal mapping of the exterior of $\Omega$ onto the exterior of the unit disk $\{w$ : $|w| \geq 1\}$. Then the vector $x_{m}$ extracted from $K_{m}\left(A ; A^{a} r_{0}\right)$ satisfies

$$
\left\|A^{a} r_{m}\right\| \leq K m^{a+2(\widehat{k}-1)} \rho^{m},
$$

for all $m$, where $K$ is a positive constant independent of $m, \widehat{k}=\max \left\{k_{j}: k_{j}=\right.$ $\left.\operatorname{ind}\left(A-\lambda_{j}\right), \lambda_{j} \in \sigma(A) \backslash\{0\}\right\}$ and $\rho=|1 / \Phi(0)|<1$.

We know the $x_{m}^{\prime} s$ produced by DGMRES is

$$
x_{m}=x_{0}+\sum_{i=1}^{m-a} c_{i} A^{a+i-1} r_{0}=x_{0}+p(A) r_{0} .
$$


Let $z_{1}, z_{2}, \cdots, z_{l}$ is a set of linear independent eigenvectors corresponding to eigenvalues $\lambda_{1}, \lambda_{2}, \cdots, \lambda_{l}$. We add some new vectors $\widehat{z}_{l+1}, \widehat{z}_{l+2}, \cdots, \widehat{z}_{n}$ such that $z_{1}, \cdots z_{l}, \widehat{z}_{l+1}, \cdots, \widehat{z}_{n}$ is a basis in $\mathbb{C}^{n}$. Then $r_{0}=b-A x_{0}=\sum_{i=1}^{l} \beta_{i} z_{i}+\sum_{i=1+1}^{n} \beta_{i} \widehat{z}_{i}$ and

$$
\begin{aligned}
& r_{m}=b-A x_{m}=b-A\left(x_{0}+p(A) r_{0}\right) \\
= & r_{0}-A p(A)\left[\sum_{i=1}^{l} \beta_{i} z_{i}+\sum_{i=l+1}^{n} \beta_{i} \widehat{z}_{i}\right] \\
= & r_{0}-\sum_{i=1}^{l} \lambda_{i} p\left(\lambda_{i}\right) z_{i}-\sum_{i=l+1}^{n} \beta_{i} A p(A) \widehat{z}_{i} \\
= & \sum_{i=1}^{l} \beta_{i} z_{i}+\sum_{i=l+1}^{n} \beta_{i} \widehat{z}_{i}-\sum_{i=1}^{l} \lambda_{i} p\left(\lambda_{i}\right) z_{i}-\sum_{i=l+1}^{n} \beta_{i} A p(A) \widehat{z}_{i} \\
= & \sum_{i=1}^{l}\left(\beta_{i} z_{i}-\lambda_{i} p\left(\lambda_{i}\right) z_{i}\right)+\sum_{i=l+1}^{n}\left(\beta_{i}-\beta_{i} A p(A)\right) \widehat{z}_{i} .
\end{aligned}
$$

Thus

$$
A^{a} r_{m}=\sum_{i=1}^{l}\left[\beta_{i} \lambda_{i}^{a} z_{i}-\lambda_{i}^{a+1} p\left(\lambda_{i}\right) z_{i}\right]+\sum_{i=l+1}^{n}\left[\beta_{i} \lambda_{i}^{a} \widehat{z}_{i}-\beta_{i} p(A) A^{a+1} \widehat{z}_{i}\right] .
$$

From Lemma 2, we know

$$
\begin{aligned}
& \left\|\sum_{i=1}^{l}\left[\beta_{i} \lambda_{i}^{a} z_{i}-\lambda_{i}^{a+1} p\left(\lambda_{i}\right) z_{i}\right]+\sum_{i=l+1}^{n}\left[\beta_{i} \lambda_{i}^{a} \widehat{z}_{i}-\beta_{i} p(A) A^{a+1} \widehat{z}_{i}\right]\right\| \\
\leq & K m^{a+2(\widehat{k}-1)} \rho^{m} .
\end{aligned}
$$

\section{DGMRES augmented with eigenvectors}

In order to accelerate the convergence of restarting GMRES, Morgan suggested some eigenvectors corresponding to a few of the smallest eigenvalues add to the Krylov subspace for GMRES. Then the convergence can be much faster. This method can be used in the process of DGMRES.

Let $k$ be the number of the eigenvectors added to the subspace. Let $r_{0}$ be the initial residual vector and $a=\operatorname{ind}(A) . K_{m}:=\operatorname{span}\left\{A^{a} r_{0}, A^{a+1} r_{0}, \cdots, A^{m-1} r_{0}\right\}$ and $K_{m, k}=\operatorname{span}\left\{A^{a} r_{0}, A^{a+1} r_{0}, \cdots, A^{m-1} r_{0}, z_{1}, z_{2}, \cdots, z_{k}\right\}$, where $z_{1}, z_{2}, \cdots, z_{k}$ are the eigenvectors added to $K_{m}$. The approximated Drazin-inverse solution will be extracted from $K_{m, k}$. That is

$$
x_{m}=x_{0}+\sum_{i=1}^{k} \alpha_{i} z_{i}+\sum_{i=1}^{m-a} c_{i} A^{a+i-1} r_{0}
$$


. which satisfies

$$
\left\|A^{a} r_{m}\right\|=\min _{x \in x_{0}+K_{m, k}\left(A ; A^{a} r_{0}\right)}\left\|A^{a}(b-A x)\right\| .
$$

Suppose $z_{1}, z_{2}, \cdots, z_{k}, \cdots, z_{l}$ is the set of linear independent eigenvectors and $z_{1}, \cdots, z_{k}, \cdots, z_{l}, \widehat{z}_{l+1}, \cdots, \widehat{z}_{n}$ is a basis of $\mathbb{C}^{n}$. So we can write

$$
r_{0}=\sum_{i=1}^{k} \beta_{i} z_{i}+\sum_{i=k+1}^{l} \beta_{i} z_{i}+\sum_{i=l+1}^{n} \beta_{i} \widehat{z}_{i},
$$

and

$$
\begin{gathered}
x_{m}=x_{0}+\sum_{i=1}^{k} \alpha_{i} z_{i}+p(A) r_{0} \\
=x_{0}+\sum_{i=1}^{k} \alpha_{i} z_{i}+p(A)\left[\sum_{i=1}^{l} \beta_{i} z_{i}+\sum_{i=l+1}^{n} \beta_{i} \widehat{z}_{i}\right] .
\end{gathered}
$$

Thus

$$
\begin{aligned}
& r_{m}=b-A x_{m} \\
= & b-A x_{0}-\sum_{i=1}^{k} \alpha_{i} \lambda_{i} z_{i}-\sum_{i=1}^{l} \lambda_{i} p\left(\lambda_{i}\right) \beta_{i} z_{i}-\sum_{i=l+1}^{n} A p(A) \beta_{i} \widehat{z}_{i} \\
= & r_{0}-\sum_{i=1}^{k} \alpha_{i} \lambda_{i} z_{i}-\sum_{i=1}^{k} \lambda_{i} p\left(\lambda_{i}\right) \beta_{i} z_{i}-\sum_{i=k+1}^{l} \lambda_{i} p\left(\lambda_{i}\right) \beta_{i} z_{i}-\sum_{i=l+1}^{n} \beta_{i} A p(A) \widehat{z}_{i}
\end{aligned}
$$

and

$$
\begin{gathered}
A^{a} r_{m}=A^{a} r_{0}-\sum_{i=1}^{k} \alpha_{i} \lambda_{i}^{a+1} z_{i}-\sum_{i=1}^{k} \lambda_{i}^{a+1} p\left(\lambda_{i}\right) \beta_{i} z_{i} \\
-\sum_{i=k+1}^{l} \lambda_{i}^{a+1} p\left(\lambda_{i}\right) \beta_{i} z_{i}-\sum_{i=l+1}^{n} \beta_{i} A p(A) \widehat{z_{i}} \\
=\sum_{i=1}^{k} \beta_{i} \lambda_{i}^{a} z_{i}+\sum_{i+1}^{l} \beta_{i} \lambda_{i}^{a} z_{i}+\sum_{i=l+1}^{n} \beta_{i} A^{a} \widehat{z}_{i} \\
\quad-\sum_{i=1}^{k} \alpha_{i} \lambda_{i}^{a+1} z_{i}-\sum_{i=1}^{k} \lambda_{i}^{a+1} p\left(\lambda_{i}\right) \beta_{i} z_{i} \\
\quad-\sum_{i=k+1}^{l} \lambda_{i}^{a+1} p\left(\lambda_{i}\right) \beta_{i} z_{i}-\sum_{i=l+1}^{n} \beta_{i} A p(A) \widehat{z_{i}} \\
=\sum_{i=1}^{k}\left[\beta_{i} \lambda_{i}^{a}-\alpha_{i} \lambda_{i}^{a+1}-\lambda_{i}^{a+1} p\left(\lambda_{i}\right) \beta_{i}\right] z_{i}
\end{gathered}
$$




$$
+\sum_{i=k+1}^{l}\left(\beta_{i} \lambda_{i}^{a}-\lambda_{i}^{a+1} p\left(\lambda_{i}\right) \beta_{i}\right) z_{i}+\sum_{i=l+1}^{n}\left[\beta_{i} A^{a}-\beta_{i} A p(A)\right] \widehat{z}_{i}
$$

Since DGMRES augmented with eigenvectors makes $\left\|A^{a} r_{m}\right\|$ minimized, we have $\alpha_{i}=\frac{\beta_{i} \lambda_{i}^{a}-\lambda_{i}^{a+1} p\left(\lambda_{i}\right) \beta_{i}}{\lambda_{i}^{a+1}}$ and

$$
A^{a} r_{m}=\sum_{i=k+1}^{l}\left(\beta_{i} \lambda_{i}^{a}-\lambda_{i}^{a+1} p\left(\lambda_{i}\right) \beta_{i}\right) z_{i}+\sum_{i=l+1}^{n}\left[\beta_{i} A^{a}-\beta_{i} A p(A)\right] \widehat{z}_{i}
$$

From Lemma 1, (4), and (7), we get the follow theorem.

Theorem 3 Denote the spectrum of $A$ by $\sigma(A)=\left\{\lambda_{1}, \lambda_{2}, \cdots, \lambda_{l}\right\} . \sigma_{a}(A):=$ $\left\{\lambda_{k+1}, \lambda_{k+2}, \cdots, \lambda_{l}\right\}(k \leq l)$. Choose $\Omega_{a}$ to be a closed domain in the $\lambda-$ plan that contains $\sigma_{a}(A) \backslash\{0\}$ but not $\lambda=0$, such that its boundary is twice differentiable with respect to arc-length. Denote by $\Phi_{a}(\lambda)$ the conformal mapping of the exterior of $\Omega_{a}$ onto the exterior of the unit disk $\{w:|w| \geq 1\}$. Then the vector $x_{m}$ generated by DGMRES augmented with eigenvectors satisfies

$$
\left\|A^{a} r_{m}\right\| \leq K m^{a+2(\widehat{k}-1)} \rho_{a}^{m},
$$

for all $m$, where $K$ is a positive constant independent of $m, \widehat{k}=\max \left\{k_{j}: k_{j}=\right.$ $\left.\operatorname{ind}\left(A-\lambda_{j}\right), \lambda_{j} \in \sigma_{a}(A) \backslash\{0\}\right\}$ and $\rho_{a}=\left|1 / \Phi_{a}(0)\right|<1$.

Compared (9) with (4), we know the convergence of DGMRES augmented with eigenvectors is faster than that of DGMRES.

The implementation of DGMRES augmented with eigenvectors is different from that of GMRES. We derive the algorithm step by step.

1. Let the initial vector $x_{0}=0$. Compute $\beta=\left\|A^{a} r_{0}\right\|$ and set $v_{1}=$ $\left(A^{a} r_{0}\right) / \beta$.

2. Orthogonalize the vectors $A^{a} r_{0}, \cdots, A^{m-1} r_{0}$ via Arnoldi-Gram-Schmidt process.

For $j=1,2, \ldots, m-a$.

For $i=1,2, \cdots, j$

Compute $h_{i j}=\left\langle A v_{j}, v_{i}\right\rangle$.

$\widehat{v_{j+1}}=A v_{j}-h_{i j} v_{i}$

end $\mathrm{i}$

Let $h_{j+1, j}=\left\|\widehat{v}_{j}\right\|$

Set $v_{j+1}=\widehat{v}_{j} / h_{j+1, j}$

end $\mathrm{j}$

Consequently, we get a orthogonal matrix $V_{m-a}=\left[v_{1}, v_{2}, \cdots, v_{m-a}\right]$ and a $(m-a+1) \times(m-a)$ Hessenberg matrix $\bar{H}^{\sharp}$.

3. Compute k approximated smallest magnitude nonzero eigenvalues of $A$ and add their corresponding eigenvectors $z_{1}, \cdots, z_{k}$ to the subspace. Let $H^{\sharp}=$ 
$\left[h_{i j}\right]_{(m-a) \times(m-a)}$. Obviously $V_{m-a}^{T} A V_{m-a}=H^{\sharp}$. When $m \leq q-1$, where $q$ is the degree of the minimal polynomial of $A$, the $\bar{H}^{\sharp}$ has full rank. We suppose $\lambda_{1}, \lambda_{2}, \cdots, \lambda_{k}$ are $\mathrm{k}$ smallest eigenvalues of $H^{\sharp}$ and $y_{1}, y_{2}, \cdots, y_{k}$ are the eigenvectors corresponding to them. Then $z_{i}:=V_{m-a} y_{i}(i=1,2, \cdots, k)$ are the approximated eigenvectors of $A$. We add them to the subspace and orthogonalize.

For $j=m-a+1, \cdots, m-a+k$

For $i=1,2, \cdots, j$

Compute $h_{i j}=\left\langle A z_{j-m+a}, v_{i}\right\rangle$.

Compute $\widehat{v}_{j+1}=A z_{j-m+a}-h_{i j} v_{i}$

end i

Set $h_{j+1, j}=\| \widehat{v}_{j+1}$

Set $v_{j+1}=\widehat{v}_{j+1} / h_{j+1, j}$

end $\mathrm{j}$.

Denote $W=\left[v_{1}, v_{2}, \cdots, v_{m-a}, z_{1}, \cdots, z_{k}\right], \bar{H}^{(0)}=\left[h_{i j}\right]_{m-a+k+1, m-a+k}$, and $V^{(0)}=\left[v_{1}, v_{2}, \cdots, v_{m-a}, v_{m-a+1}, \cdots, v_{m-a+k+1}\right]$. It is easy to see $A W=V^{(0)} \bar{H}^{(0)}$ and $x_{m}=x_{0}+W y_{m}$ for some $y_{m} \in \mathbb{C}^{n \times n}$. It follows that $r_{m}=r_{0}-A W y_{m}=$ $r_{0}-V^{(0)} \bar{H}^{(0)} y_{m}$. So we get

$$
A^{a} r_{m}=A^{a} r_{0}-A^{a+1} W y_{m}=\beta v_{1}-A^{a+1} W y_{m} .
$$

From this, we should continue to deal with $A^{a+1} W$.

$$
\begin{aligned}
A^{a+1} W & =A^{a}(A W)=A^{a} V^{(0)} \bar{H}^{(0)} \\
& =A^{a-1}\left(A V^{(0)}\right) \bar{H}^{(0)} \\
& =A^{a-1}\left[v_{1}, v_{2}, \cdots, v_{m-a}, v_{m-a+1}, v_{m-a+2}, \cdots, v_{m-a+k+2}\right] \bar{H}^{(1)} \bar{H}^{(0)} \\
& =A^{a-1} V^{(1)} \bar{H}^{(1)} \bar{H}^{(0)} \\
& \vdots \\
& =A^{a-(a-1)} V^{(a-1)} \bar{H}^{(a-1)} \cdots \bar{H}^{(0)} \\
& =V^{(a)} \bar{H}^{(a)} \bar{H}^{(a-1)} \cdots \bar{H}^{(0)}
\end{aligned}
$$

Denote $\bar{H}^{(a)} \cdots \bar{H}^{(0)}$ by $\bar{H}$. From the above, we get

$$
A^{a+1} W=V^{(a)} \bar{H} .
$$

Since $r_{m}$ is the residual vector produced by DGMRES augmented with eigenvectros, from (9) and (10), we have

$$
\begin{aligned}
\left\|A^{a} r_{m}\right\| & \left.=\beta v_{1}-A^{a+1} W y_{m}\|=\| \beta v_{1}-V^{(a)} \overline{(} H\right) y_{m} \| \\
& =\left\|\beta e_{1}-\bar{H} y_{m}\right\|=\min _{y}\left\|\beta e_{1}-\bar{H} y\right\|
\end{aligned}
$$

Apply the QR factorization of $\bar{H}: \bar{H}=Q R$, where $R$ is upper triangular. Thus $y_{m}$ satisfies $R y_{m}=\beta\left(Q^{T} e_{1}\right)$ and $x_{m}$ follows. 
4. The practical computation of $\bar{H}$. From the above we know

$$
A\left[v_{1}, \cdots, v_{m-a}, z_{1}, \cdots, z_{k}\right]=\left[v_{1}, \cdots, v_{m-a}, v_{m-a+1}, \cdots, v_{m-a+k+1}\right] \bar{H}^{(0)},
$$

and

$$
\begin{aligned}
& A\left[v_{1}, v_{2}, \cdots, v_{m-a}, v_{m-a+1}, \cdots, v_{m-a+k+1}\right. \\
= & {\left[v_{1}, v-2, \cdots, v_{m-a}, v_{m-a+1}, v_{m-a+2}^{(1)}, \cdot, v_{m-a+k+1}^{(1)}\right] \bar{H}^{(1)} }
\end{aligned}
$$

where

$$
\bar{H}^{(1)}=\left(\begin{array}{ccccccc}
h_{11} & h_{12} & \cdots & h_{1, m-a} & \widehat{h}_{1, m-a+1} & \cdots & \widehat{h}_{1, m-a+k+1} \\
h_{21} & h_{22} & \cdots & h_{2, m-a} & \widehat{h}_{2, m-a+1} & \cdots & \widehat{h}_{1, m-a+k+1} \\
0 & h_{32} & \cdots & h_{3, m-a} & \widehat{h}_{3, m-a+1} & \cdots & \widehat{h}_{3, m-a+k+1} \\
& \cdots & \cdots & & \widehat{h}_{m-a+1, m-a+1} & \cdots & \widehat{h}_{m-a+1, m-a+k+1} \\
0 & 0 & \cdots & h_{m-a+1, m-a} & \widehat{h}_{m-a+2, m-a+1} & \cdots & \widehat{h}_{m-a+2, m-a+k+1} \\
0 & 0 & \cdots & 0 & & & \widehat{h}_{m-a+k+2, m-a+k+1}
\end{array}\right)
$$

$\widehat{h}_{i j}$ is the new entries we need to compute when from $\bar{H}^{(0)}$ to $\bar{H}^{(1)}$, that is, we need to compute $\frac{[2(m-a+2)+k] \times(k+1)}{2}$ entries additional.

Next

$$
\begin{aligned}
& A\left[v_{1}, v_{2}, \cdots, v_{m-a}, v_{m-a+1}, v_{m-a+2}^{(1)}, \cdots, v_{m-a+k+1}^{(1)}\right] \\
= & {\left[v_{1}, \cdots, v_{m-a+1}, v_{m-a+2}^{(1)}, v_{m-a+3}^{(2)}, \cdots, v_{m-a+k+2}^{(2)}\right] \bar{H}^{(2)} . }
\end{aligned}
$$

where

$$
\bar{H}^{(1)}=\left(\begin{array}{ccccccc}
h_{11} & h_{12} & \cdots & h_{1, m-a+1} & \widehat{h}_{1, m-a+2} & \cdots & \widehat{h}_{1, m-a+k+2} \\
h_{21} & h_{22} & \cdots & h_{2, m-a+1} & \widehat{h}_{2, m-a+2} & \cdots & \widehat{h}_{2, m-a+k+2} \\
0 & h_{32} & \cdots & h_{3, m-a+1} & \widehat{h}_{3, m-a+2} & \cdots & \widehat{h}_{3, m-a+k+2} \\
& & \vdots & \vdots & & & \\
0 & 0 & \cdots & h_{m-a+2, m-a+1} & \widehat{h}_{m-a+2, m-a+2} & \cdots & \widehat{h}_{m-a+2, m-a+k+2} \\
& & \vdots & \vdots & & & \\
0 & 0 & \cdots & 0 & 0 & \cdots & \widehat{h}_{m-a+k+2, m-a+k+2}
\end{array}\right)
$$

Similarly, from $\bar{H}^{(1)}$ to $\bar{H}^{(2)}$, there are $\frac{[2(m-a)+5+k] \times(k+1)}{2}$ need to be computed. Following this way, we can continue to get $\bar{H}^{(3)}, \cdots, \bar{H}^{(a)}$ and $\bar{H}$ can be computed.

We summarize the above as the following algorithm.

Algorithm 4 DGMRES augmented with eigenvectors

1. Pick initial vector $x_{0}$ and compute $r_{0}=b-A x_{0}$ and $\beta=\left\|A^{a} r_{0}\right\|$.

2. Apply Arnoldi process to $A^{a} r_{0}, \cdots, A^{m-1} r_{0}$. 


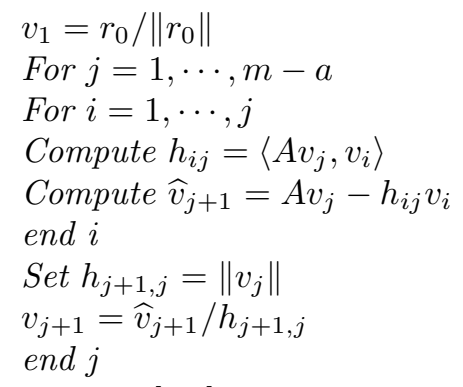

4. Denote $\left[h_{i j}\right]_{(m-a) \times(m-a)}$ by $H$ and compute its $k$ eigenvectors $y_{1}, \cdots, y_{k}$ corresponding to $k$ smallest magnitude eigenvalues. Compute $z_{i}=V y_{i}$. We add $z_{i}^{\prime} s$ to the subspace and denote $W=\left[v_{1}, \cdots, v_{m-a}, z_{1}, \cdots, z_{k}\right]$. We apply Arnoldi process to $A W$ and get the matrix $V^{(0)}$ and $\bar{H}^{(0)}$.

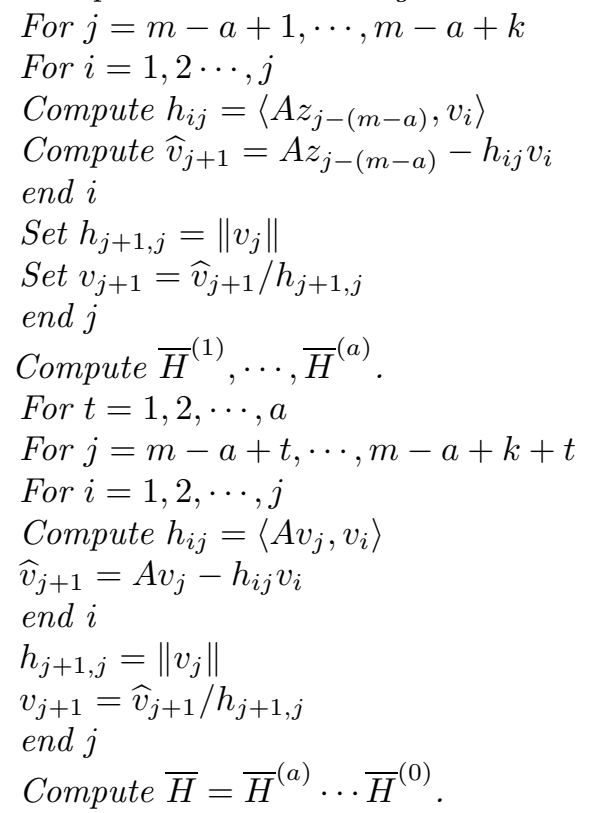

6. Compute the $Q R$ factorization of $\bar{H}: \bar{H}=Q R$, where $R$ is upper triangular. Set $c=\beta Q^{T} e_{1}$. Solve the least-square problem $R_{m} y_{m}=\beta\left(Q^{T} e_{1}\right)$ and $x_{m}=$ $x_{0}+W y_{m}$. If $r_{m}:=b-A x_{m}$ satisfies the residual norm then stop; else set $x_{0}=x_{m}$ and go to 2 .

\section{Numerical examples}

For convenience, We denote the DGMRES augmented with eigenvectors by ADGMRES. In this section we present some numerical examples to compare ADGMRES to DGMRES. All the experiments were performed in $M A T L A B{ }^{\circledR}$ 7.5 on an Inter Core 2 Duo $2000 \mathrm{MHz}$ PC with main memory 1000M. 
Example 1 We take $A$ to be a 12 by 12 singular matrix which has the following Jordan canonical form

$$
\left(\begin{array}{llllllllllll}
1 & 1 & 0 & 0 & 0 & 0 & 0 & 0 & 0 & 0 & 0 & 0 \\
0 & 1 & 1 & 0 & 0 & 0 & 0 & 0 & 0 & 0 & 0 & 0 \\
0 & 0 & 1 & 0 & 0 & 0 & 0 & 0 & 0 & 0 & 0 & 0 \\
0 & 0 & 0 & 3 & 1 & 0 & 0 & 0 & 0 & 0 & 0 & 0 \\
0 & 0 & 0 & 0 & 3 & 1 & 0 & 0 & 0 & 0 & 0 & 0 \\
0 & 0 & 0 & 0 & 0 & 3 & 0 & 0 & 0 & 0 & 0 & 0 \\
0 & 0 & 0 & 0 & 0 & 0 & 7 & 0 & 0 & 0 & 0 & 0 \\
0 & 0 & 0 & 0 & 0 & 0 & 0 & 8 & 0 & 0 & 0 & 0 \\
0 & 0 & 0 & 0 & 0 & 0 & 0 & 0 & 9 & 1 & 0 & 0 \\
0 & 0 & 0 & 0 & 0 & 0 & 0 & 0 & 0 & 9 & 0 & 0 \\
0 & 0 & 0 & 0 & 0 & 0 & 0 & 0 & 0 & 0 & 0 & 1 \\
0 & 0 & 0 & 0 & 0 & 0 & 0 & 0 & 0 & 0 & 0 & 0
\end{array}\right)
$$

The right side $b=(1,1, \cdots, 1)^{T}$. The ADGMRES uses $m=6, k=1$ and DGMRES uses $m=7$ and denote them by DGMRES(6,1), DGMRES(7) respectively. So they use the same size subspace. When $\frac{\left\|A^{a} r_{m}\right\|}{\left\|A^{a} b\right\|}<\epsilon$, we stop. The convergence curve for ADGMRES and DGMRES are indicated in Fig 1. It is easy to see ADGMRES convergence faster than DGMRES

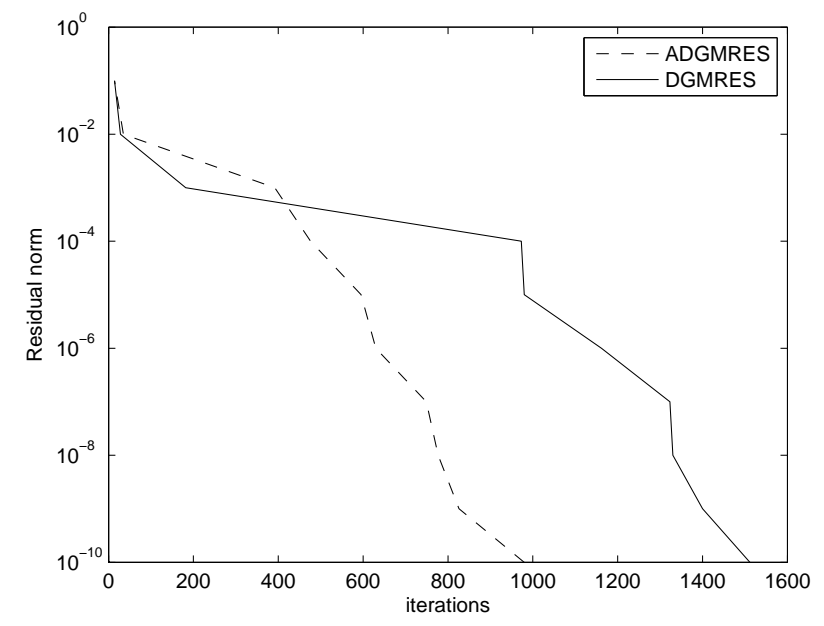

Fig1. The convergence curves for ADGMRES(6,1) and DGMRES(7)

Example 2. In this example the matrix $A$ is the same as the above but let $a_{7,7}=1000$. Obviously such $A$ has a larger eigenvalue 1000 . We expect ADGMRES $(4,1)$ convergence much faster than DGMRES(5). As indicated in Fig 2., it is the case. 


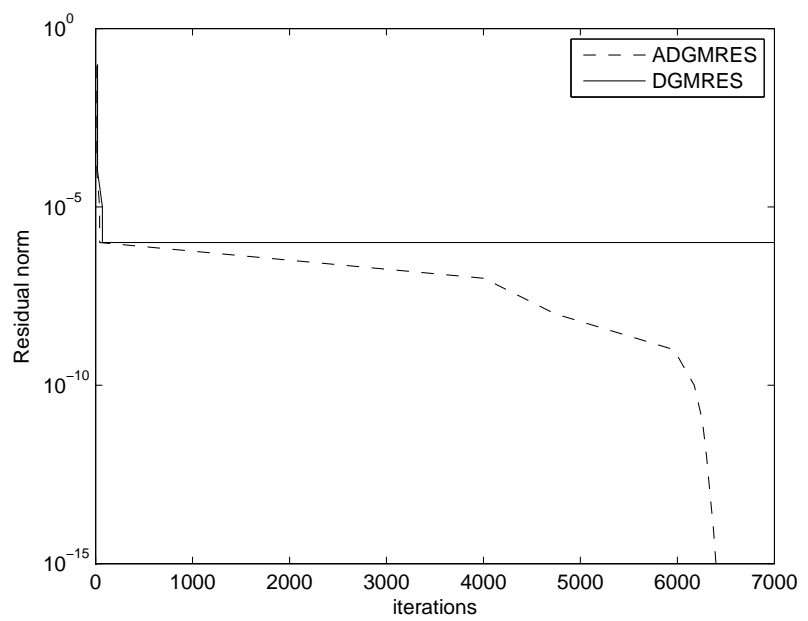

Fig2. The convergence curves for ADGMRES(4,1) and DGMRES(5) when $A$ has a very lager eigenvalue.

From Fig 2. we can see after 70 iterations the DGMRES(5) stagnates but after 5000 iterations the curve of $\operatorname{ADGMRES}(4,1)$ decreases steeply.

Example 3. In this example we take $a_{77}=0.001$, that is, $A$ has a very small eigenvalue. From Fig 3. we see DGMRES(5) stagnates after 50 iterations but ADGMRES $(4,1)$ still works well.

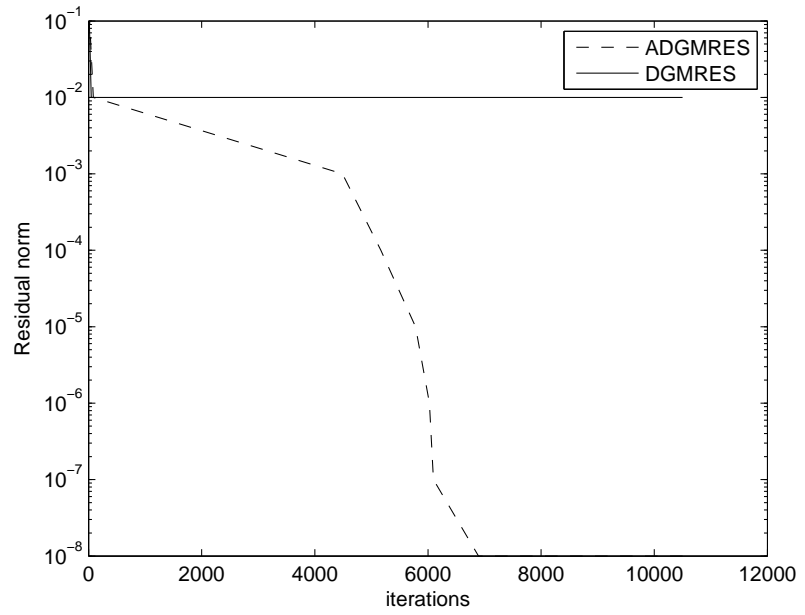

Fig3. The convergence curves for ADGMRES(4,1) and DGMRES(5) when $A$ has a very small eigenvalue. 
Example 4. This example comes from [5].

$$
A=\left(\begin{array}{llll}
1 & 1 & 1 & 2 \\
0 & 1 & 3 & 4 \\
0 & 0 & 1 & 1 \\
0 & 0 & 0 & 0
\end{array}\right), b=\left(\begin{array}{c}
-4 \\
7 \\
1 \\
0
\end{array}\right)
$$

In [5] The author found that DGMRES(2) converges faster than DGMRES(3). Since $A$ has only one nonzero eigenvalue 1, we infer from Theorem that the convergence of $\operatorname{ADGMRES}(2,1)$ is as the same as that of DGMRES(2). From Table 1 we observe it is the case. At the same time we also see DGMRES(3) stagnates. The residual norm produced by the three methods is indicated in the following table.

\begin{tabular}{c|ccc} 
runs & ADGMRES $(2,1)$ & DGMRES(3) & DGMRES $(2)$ \\
\hline 50 & 0.0038 & 0.00279 & 0.0038 \\
100 & $1.23 \times 10^{-5}$ & 0.00276 & $1.23 \times 10^{-5}$ \\
200 & $1.71 \times 10^{-9}$ & 0.00276 & $1.72 \times 10^{-9}$ \\
300 & $6.155 \times 10^{-14}$ & 0.00276 & $6.145 \times 10^{-14}$
\end{tabular}

Table 1. The residual norm produced by ADGMRES(2,1), DGMRES(3), DGMRES(2) after 50, 100, 200, 300 runs respectively.

\section{Conclusion.}

The DGMRES method augmented with eigenvectors can improve the convergence, especially when the matrix has small or large eigenvalues. The DGMRES method often stagnates (see [4, [5]). The DGMRES augmented with eigenvectors is a good choice to overcome the stagnation. When $k$, the number of the eigenvectors added to the subspace, is large, the method is expensive. So in practical use we usually choose a small $k$.

\section{References}

[1] R.Morgan, A restarted GMRES method augmented with eigenvectors, SIAM J.Matrix Anal.Appl. 16 (1995)1154-1171

[2] A.Sidi, A unified approach to Krylov subspace methods for the Drazininverse solution of singular nonsysmmetric linear systems, Lin. Alg. Appl., 298 (1999) 99-113

[3] A.Sidi, DGMRES: A GMRES-type algorithm for Drazin-inverse solution of singular nonsysmmetric linear systems, Lin. Alg. Appl., 335 (2001) $189-204$

[4] J.Zhou, Y.Wei, Stagnation analysis of DGMRES, Appl. Math. Comp., 151 (2004) 27-39 
[5] J.Zhou, Y.Wei, The analysis of restart DGMRES for solving singular linear systems, Appl. Math. Comp., 176 (2006) 293-301 